\title{
Sur trois pointes de javelot en fer d'un tumulus du Maroc Oriental
}

\author{
GeORGES SOUVILLE*
}

\begin{abstract}
RÉSUMÉ ABSTRACT
Dans un tumulus du Maroc oriental, formé d'une enceinte circulaire avec des bastions accolés, ont été recueillies trois pointes de javelot en fer. Celles-ci présentent une ornementation élaborée et Three heads of iron javelin came from an oriental morrocan barrow formed by a circular enclosure. They presented an elaborated unusual ornament; the sharp end was deliberately turned over.
\end{abstract} inhabituelle; la pointe a été retournée

intentionnellement.

Autour de 1950, un officier, M. Robert Biard, alors capitaine, a fouillé un tumulus au Maroc oriental, à Gaidet el Felles, à environ $11 \mathrm{~km}$ au S.O. de Berguent ${ }^{1}$. Simplement signalé (R. Thouvenot, 1951-52: 149), ce monument n'a donné lieu à aucune publication complète.

II s'agissait d'une large enceinte en pierres sèches de $4 \mathrm{~m}$ de diamètre et plus d'1,5 m de haut, avec trois "bastions" semi-elliptiques accolés à cette enceinte. On ne trouva pas de restes humains et le mobilier se réduisait à trois pointes de fer qui représentent cependant l'intérèt principal de cette découverte.

* Antiquités africaines, C.N.R.S., Aix-en-Provence. Les figures 1 et 2 ont été mises au net par E. Lamouroux; les figures 3,4 et 5 sont dues à J. Lenne. La figure 6 est un cliché d'A. Chéné, Centre Camille Jullian, C.N.R.S., Aix-en-Provence. Je leur adresse à tous les trois mes bien vifs remerciements.

1 Carte du Maroc au 1/200000, Berguent, C.L. environ $282 \times 802$. 


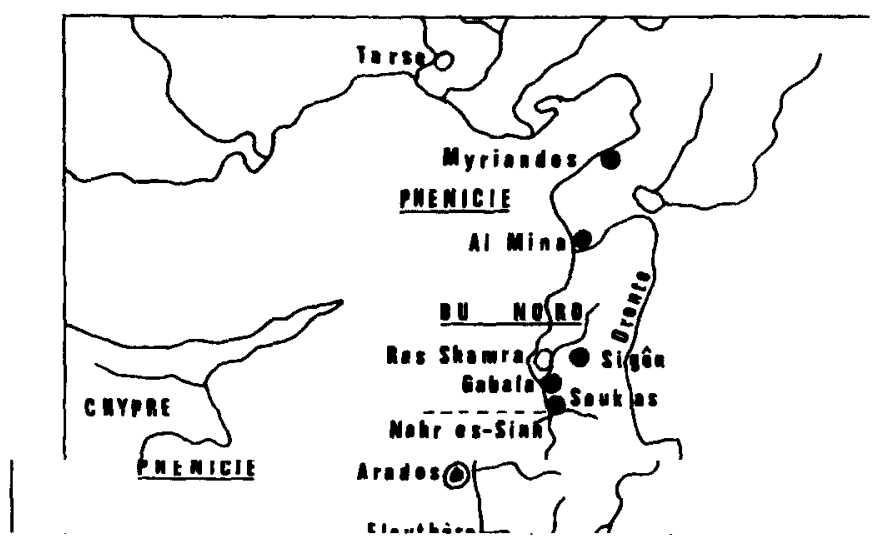

Fig. 1. Tumulus à antenne d'Oujda (d'après L. Voinot, 1910:524, pl. X, 7).

La description de ce monument rappelle celle de nombreux tumulus fouillés ou reconnus par $L$. Voinot $(1910,1913,1916)$ dans la même région: gros blocs irréguliers formant une sorte d'enceinte avec embryons de branches (fig. 1) que l'auteur (1910: 526) rapprochait avec raison de monuments sahariens (G. Camps, 1965: 481 - G. Souville, 1965: 483-485 et 1968: 52-53).
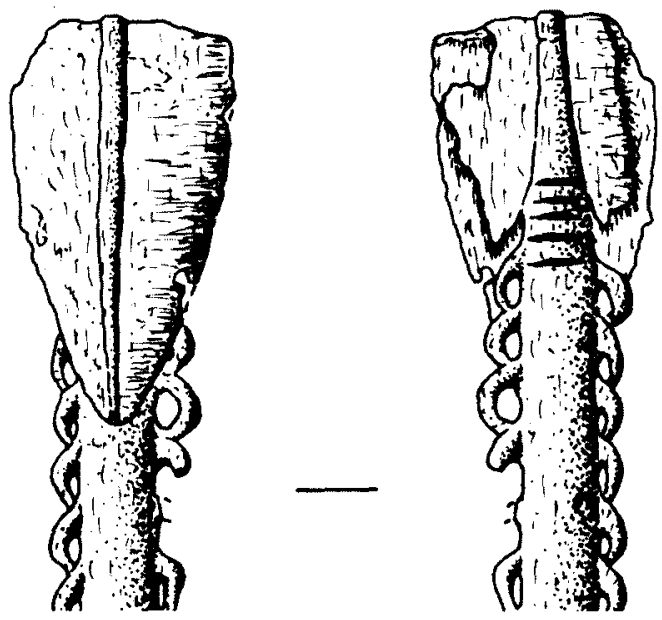

Fig. 2. Fer de javelot recourbé trouvé dans un tumulus de la région d'Oujda (d'après $L$. Voinot, $1910: 521, \mathrm{pl}$. XI, 1-3). 
La présence de trois pointes de javelot en fer est particulièrement intéressante. Les fers de javelot sont inexistants en Tunisie et en Algérie orientale mais relativement fréquents en Algérie occidentale et au Maroc, notamment au Maroc oriental, au Tafilalet (G. Camps, 1963: 443-444) mais aussi au Moyen Atlas, à Tayadirt (N. Lambert et G. Souville, 1966: 227 - N. Lambert, 1967: 249) et dans le Nord marocain (M. Ponsich, 1967 et 1968: 62 - G. Camps, 1976: 44). Leur existence n'est pas surprenante. Le javelot à manche court était l'arme de prédilection des Numides et des Maures (S. Gsell, 1929: 49). Ces armes légères étaient lancées de loin et pouvaient être tenues à deux ou trois dans la même main (G. Camps, 1963: 434). Ces pointes de javelot sont d'un type élémentaire, avec un emmanchement par une douille fendue de très petit diamètre $(0,015 \mathrm{~m}$ à Oujda) et ornées d'une nervure centrale (G. Camps, 1963: 435).

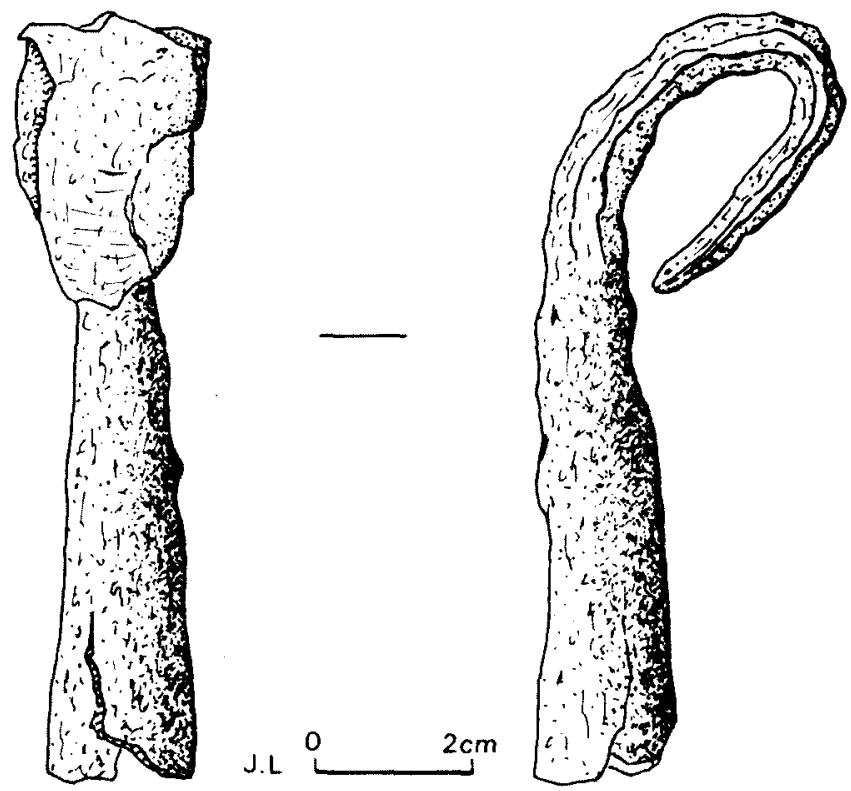

Fig. 3. Fer de javelot de la région de Berguent (non restauré).

Leur fréquence au Maghreb occidental est sans doute d'origine rituelle, cette arme n'étant pas plus répandue chez les Maures que chez les Numides. Cet aspect cultuel est renforcé par le fait que ces fers de javelot ont la pointe recourbée intentionnellement. C'est le cas à Oujda (fig. 2) 
comme ici, selon un usage très fréquent au Maghreb occidental (G. Camps, 1953: 435, note 2) mais également en Europe à La Tène II (J. Déchelette, 1914: 1149).

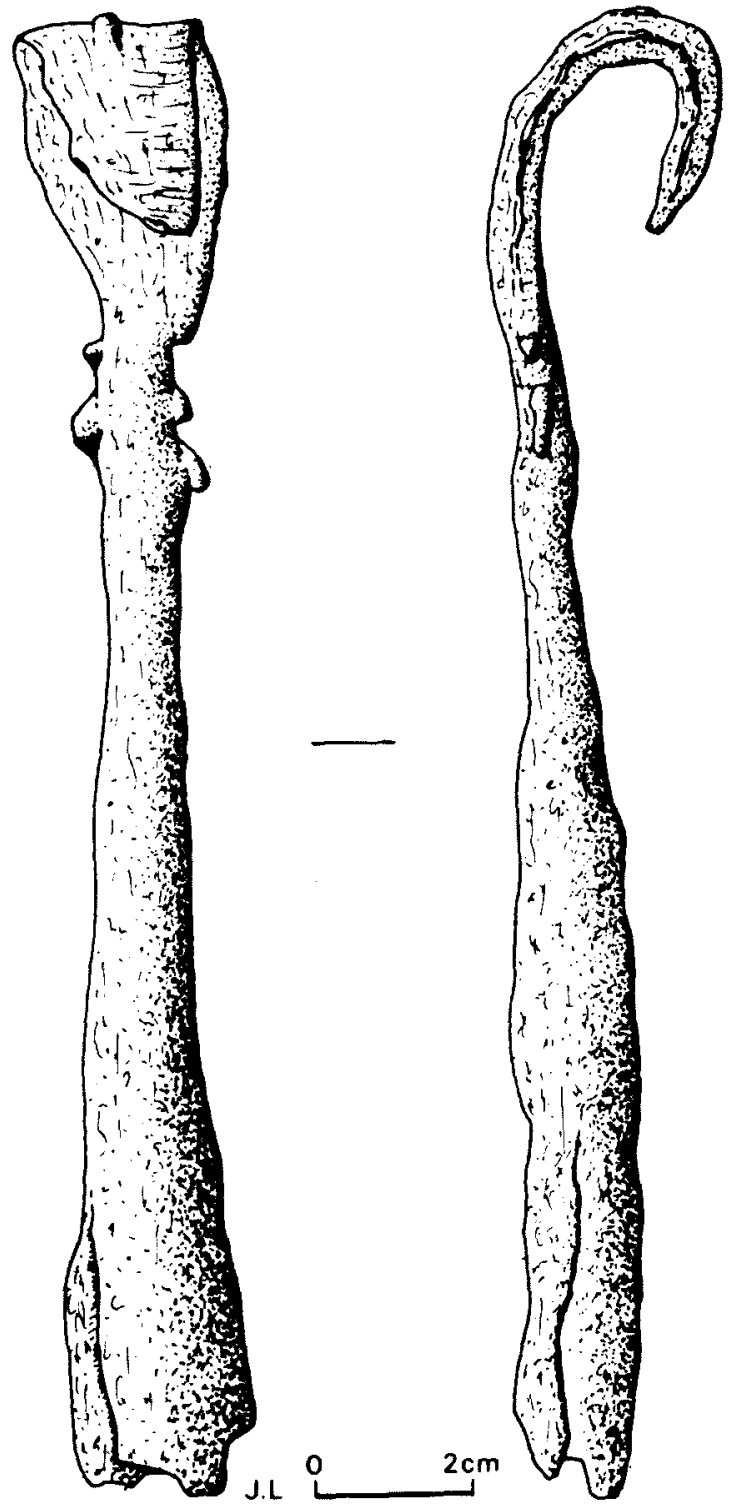

Fig. 4. Fer de javelot de la région de Berguent (non restauré). 
Sur ces trois fers de javelot, un seul a pu être restauré; les deux autres (fig. 3 et 4 ) sont très altérés par l'oxydation et par leur séjour en terre. Leurs décors toutefois sont comparables à ceux de la pièce restaurée ${ }^{2}$. Par leur ornementation comme par le soin dans l'exécution, les trois objets sont nettement plus élaborés qu'à Oujda.

Un des fers de javelot a été restauré par les soins du laboratoire d'archéologie des métaux de Jarville. Il a des dimensions comparables aux deux autres ${ }^{3}$. Un prélèvement effectué sur la douille "révèle un fer doux, non carburé, assez pur malgré la présence de scories incluses ou dissoutes. Il s'agit donc d'un métal obtenu au bas foyer, bien forgé et sans surchauffe réelle (phase de finition à température modérée).
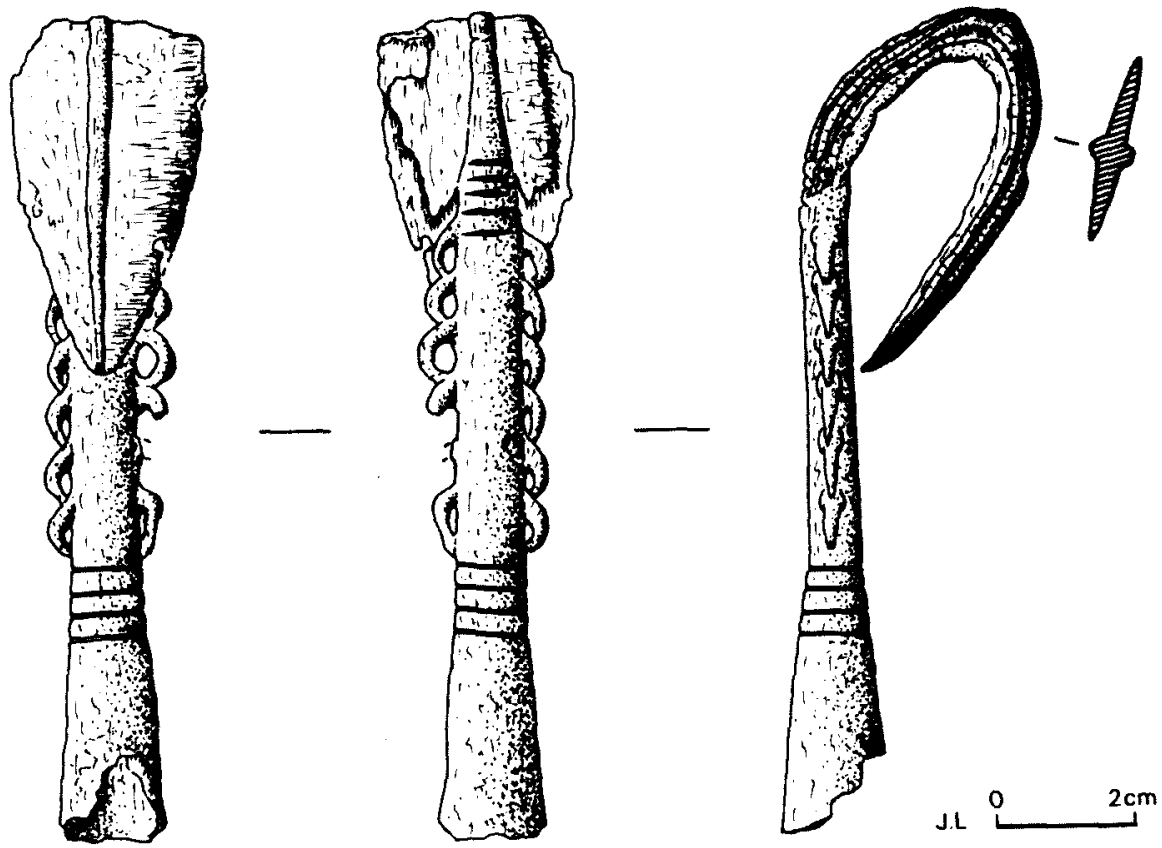

Fig. 5. Fer de javelot de la région de Berguent (après restauration).

2 L'un pèse $50 \mathrm{~g}$, mesure $9,4 \mathrm{~cm}$ de long et 1,8 au diamètre de la douille ; l'autre, respectivement $72,5 \mathrm{~g}, 18,5 \mathrm{~cm}$ et $2,2 \mathrm{~cm}$.

3 Poids: $69 \mathrm{~g}$, longueur: $12 \mathrm{~cm}$, diamètre de la douille: $1,3 \mathrm{~cm}$; mesures prises après restauration. 


$$
p
$$


Une nervure centrale en relief occupe toute la longueur de la pointe recourbée. Des cercles parallèles sont gravés soigneusement à la base de la pointe et sur la partie proche de la douille. On retrouve ces cercles mais en plus grand nombre sur la pointe d'Oujda (fig. 2). II y a en outre, ce qui est plus exceptionnel, de chaque côté, six oeillets disposés régulièrement; sur un bord, deux oeillets sont brisés (fig. 5 et 6 ).

Par son décor très élaboré, cette pointe est tout à fait exceptionnelle. On ne peut trouver rien d'équivalent au Maghreb et sans doute dans le reste de l'Afrique.

\section{BIBLIOGRAPHIE}

CAMPS, G., 1961: Aux origines de la Berbérie. Monuments et rites funéraires protohistoriques, Paris.

- 1965: Essai de classification des monuments protohistoriques de l'Afrique du Nord. Bull. Soc. préhist. franç., 62, 476-481.

- 1976: Nouvelles observations sur l'âge du fer indigène en Afrique du Nord. L'âge du fer en Méditerranée, Ajaccio, (1976), 37-48.

DÉCHELETTE, J., 1914: Manuel d'archéologie préhistorique, celtique et gallo-romaine, II, 3. Paris.

GSELL, S., 1929: Histoire ancienne de l'Afrique du Nord, 6, Les royaumes indigènes, Paris, $2 e$ éd. LAMBERT, N., 1967: Tayadirt, une nécropole en Haute-Moulouya. Libyca, Anthr.-Préhist.-Ethn., 15, 215-260.

Lambert, N. et Souville, G., 1966: La nécropole de Tayadirt (Moyen Atlas marocain). Note préliminaire. Congr. préhist. France, C.-R. $18 \mathrm{e}$ session, Ajaccio, 1966 (Paris, 1966), 217-229.

Ponsich, M., 1967: Nécropoles phéniciennes de la région de Tanger. Tanger, 66, 72, 75, 79,87, $117,138,159,167,207,219$.

- 1968: Nécropoles puniques de la région de Tanger. $91^{\circ}$ Congr. nat. Soc. savantes, Rennes, 1966 (Paris, 1968), 55-69.

SOUVILLE, G., 1965: Eléments nouveaux sur les monuments funéraires préislamiques du Maroc. Bull. Soc. préhist. franç., 62, 482-493.

- 1968: Los monumentos funerarios preislámicos de Marruecos. Ensayo de clasificación y distribución. Ampurias, 30, 39-61.

Thouvenot, R., 1951-1952: Rapport sur l'activité du service des antiquités du Maroc en 1951. Bull. archéol. Comité Trav. hist. et sci, 142-157.

VoInot, L., 1910: Les tumuli d'Oudjda. Bull. Soc. Géogr. et Archéol. Oran, 30, 516-528.

- 1913: Note sur les tumuli et quelques vestiges d'anciennes agglomérations de la région d'Oudja, Ibid., 33, 507-527.

- 1916: Note sur les tumuli et quelques ruines des environs d'El Aïoun Sidi Mellouk (Maroc oriental). (bid., 36, 257-277. 


\section{y baños ceremoniales. \\ Esta civilización decae hacia el siglo xvI a.C., $y$ es sustituida por nuevas culturas organizadas en simples pueblos rurales.}

\section{INTRODUCCIÓN}

Se ha dicho que la India es un "país de aldeas", e incluso en la actualidad la mayoría de los habitantes de la India viven en aldeas ${ }^{1}$. Un 85 por ciento de la población habita en las aproximadamente 600.000 aldeas, la mayor parte de las cuales no llegan a mil habitantes. Unos 204 millones de indios viven en núcleos urbanos distribuidos entre unas 4.000 ciudades y poblaciones importantes (número relativamente reducido para un país $\tan$ extenso).

Al igual que las personas que viven en ellas, las aldeas de la India varian enormemente en apariencia de una región a otra, desde la sólidas viviendas de piedra del Himalaya hasta las cabañas de bambú y esteras de las regiones cálidas del centro y el sur. Las variaciones también se dan en lo que atañe a dimensiones y disposición, desde los asentamientos dispersos alrededor de granjas aisladas a las comunidades compactas edificadas en terrenos más extensos.

Las distintas clases de moradas hindúes están compuestas de elementos cuyos paralelos se encuentran, por un lado, en la casa aplanada iraniana, y, por otra, en las chozas de los nativos de la India e Indochina. A menudo parece adivinarse también cierta relación con el tipo de vivienda de la cultura del valle del Indo, pero todavía no existen pruebas concluyentes.

Hoy en día, la ciudad típica de la India presenta tres zonas diferenciadas. En primer término se halla el distrito comercial y residencial superior, con viviendas de sólida construcción, modernos edificios de oficinas y tiendas bien abastecidas, en amplias calles repletas de gente vestida al estilo occidental. En Bombay y Calcuta, antiguos baluartes del colonialismo británico, esta zona ocupa el antiguo "barrio europeo».

\footnotetext{
La vida de los antiguos hindúes se desarrolló en tres ambientes muy diferentes: en las aldeas, en los bosques y selvas y en las ciudades. Ver H. BOEKHOFF y F. WINZER, Historia de la cultura oriental, Labor, Madrid, 1968, págs. 262-265.
} 